Rev. Elev, Méd. vét. Pays trop., 1975, 28 (2) : 137-142

\title{
Inhibition du développement des larves de Cooperia punctata et de Cooperia|pectinata chez le zébu nigérien
}

\author{
par M. GRABER $\left({ }^{*}\right)$ et P. TAGER-KAGAN $\left({ }^{* *}\right)$
}

\begin{abstract}
RESUME,
Les auteurs signalent l'existence, à la fin de la saison sèche, de larves $\mathrm{L} A$ inhibées de Cooperia punctata et de Cooperia pectinata dans l'intestin grêle d'un peu plus de 50 p. 100 des zébus sacrifiés dans la région de Niamey (République du Niger).

Ces larves reprennent leur évolution normale dès l'apparition des premières pluies.

Les auteurs, après avoir recensé les cas similaires actuellement connus en Afrique, envisagent les conséquences de cette situation sur la santé des animaux et sur le traitement des strongyloses en milieu africain.
\end{abstract}

On sait aujourd'hui que certains Nématodes sont capables, lorsqu'ils sont encore au stade larvaire $\left(L_{4}\right.$ ou $\left.L_{5}\right)$ dans l'appareil digestif ou dans l'appareil pulmonaire de leur hôte, de subir des retards ou des arrêts de croissance plus ou moins longs qui, dans les pays tempérés des deux hémisphères, se produisent généralement au cours de l'automne et de l'hiver.

Ce phénomène d'inhibition a été décrit chez le bœuf, le mouton, le porc et les parasites en cause sont Ostertagia ostertagi et Ostertagia spp. $(1,3,4,5,8,13,29,31,32)$, Nematodirus spp. $(29,32)$, Chabertia ovina (12), Haemonchus contortus $(6,7,9,10)$, Mecistocirrus digitatus (15), Cooperia oncophora $(22,27,32)$, Cooperia pectinata $(21,22)$, Cooperia spp. (8), Dictyocaulus viviparus (16) et Hyostrongylus rubidus du porc (11).

En Afrique tropicale où l'infestation des

Institut d'Elevage et de Módecine vétérinaire des Pays tropicaux, 10, rue Pierre Curie, 94700 MaisonsAlfort, France.

(*) Chaire de Parasitologie, Ecole Nationale Vétérinaire, 2, quai Chauveau, 69337 Lyon Cedex 1.

(**) Laboratoire de l'Elevage, B.P. 485, Niamey, République du Niger. ruminants est à base de Bunostomum, Gaigeria, Oesophagostomum, Cooperia, Haemonchus, plus rarement de Trichostrongylus et d'Impalaia, le phénomène signalé çà et là à plusieurs reprises $(18,20,30)$, n'a, cependant, pas fait l'objet d'études systématiques.

Aussi, a-t-il paru intéressant de relater une observation faite à l'occasion d'essais thérapeutiques entrepris au Laboratoire de l'Elevage de Niamey (République du Niger), observation qui concerne Cooperia pectinata et Cooperia punctata.

\section{MATERIEL ET METHODE}

\section{Les animaux}

75 zébus - 61 mâles et 14 femelles - ont été utilisés au cours de cette expérience. Leur poids variait de $83 \mathrm{~kg}$ pour les plus jeunes (9 mois) à $256 \mathrm{~kg}$ pour les plus âgés.

Ils avaient été achetés, en mai-juin 1974, autour de Niamey, sur les marchés du fleuve Niger, dans une région où les conditions climatiques (pluviométrie, température, degré hy- 
grométrique), l'état des pâturages et les méthodes d'élevage sont assez semblables, ce qui laissait supposer, dès le départ, que l'on aurait affaire à un parasitisme assez homogène.

Début juillet, les animaux ont été placés dans des conditions d'existence telles qu'aucune infestation ou réinfestation, en cours d'expérience, n'était matériellement possible (19).

\section{Méthode}

Chaque animal a été mis en observation du 3 au 6 juillet 1974. Durant cette période, ont été effectués simultanément :

- Des examens coproscopiques (méthode multivalente d'enrichissement par sédimentation);

- Des coprocultures en boîtes de Pétri sur papier buvard, évoluant à la température du laboratoire.

La diagnose des ceufs et des larves $L_{3}$ a été faite selon, les critères adoptés par GEVREY (16).

69 animaux, sur les 75 choisis, hébergeaient des Cooperia pectinata et des Cooperia puncta$t a$, seuls ou associés. Le niveau de l'infestation était, dans l'ensemble, faible.

Ils ont été répartis en cinq lots de quinze rigoureusement équilibrés (poids, âge, importance du parasitisme). Les deux premiers (Lots A et B) ont reçu 80 et $100 \mathrm{mg} / \mathrm{kg}$ de Thiabendazole, le troisième et le quatrième (Lots C et D) du Tartrate de Morantel à 5 et $7,5 \mathrm{mg} / \mathrm{kg}$, le demier (Lot $\mathrm{E}$ ) a servi de témoin. Le lot $\mathrm{A}$ comprenait 13 animaux porteurs de Cooperia, le lot B, 15; le lot C, 14; le lot D, 13; et le lot E, 14.

Après traitement, jusqu'à l'abattage, il a été procédé à des examens coproscopiques réguliers et à des coprocultures. En outre, les trois premiers jours, les crottes ont été ramassées plusieurs fois dans la journée, broyées dans un filet d'eau et soigneusement examinées, de manière à rechercher les Cooperia expulsés après administration des médicaments.

Passé ce délai, les animaux ont été sacrifiés à des dates variables, du 8 au 21 juillet 1974 pour les témoins et du 15 au 20 juillet 1974 pour les traités (par abattage, de 5 à 12 animaux appartenant à tous les lots).
Ils ont été autopsiés selon les techniques classiques (14).

La muqueuse duodénale, en particulier, a été grattée profondément sur une longueur de $30 \mathrm{~cm}$ (2 à 6 prélèvements par animal). L'examen au microscope du produit de raclage, placé entre lame et lamelle, permet de mettre en évidence les Cooperia ayant résisté au traitement. Ceux-ci ont été isolés, déterminés et comptés.

\section{RESULTATS}

\section{Cooperia adultes et mûrs}

Comme il a été dit plus haut, 69 animaux hébergeaient des Cooperia adultes dont les cufs, en coprocultures, évoluent en donnant des larves $L_{3}$ caractérisées par la présence de corps ovalaires réfringents situés au début de l'œsophage, de part et d'autre de celui-ci.

55 d'entre eux ont été traités au Thiabendazole et au Tartrate de Morantel. A l'autopsie, il ne restait plus aucun parasite appartenant à cette classe d'âge, ce que confirment, par ailleurs, les résultats fournis par les examens coproscopiques et les coprocultures. Les Cooperia adultes sont, en général, digérés au cours de leur transit dans le tube digestif. Quelquesuns seulement parviennent à l'extérieur et sont retrouvés intacts dans les fèces le deuxième jour faisant suite au traitement. Quant aux œufs, leur émission cesse au bout de 72 heures.

Chez les 14 témoins, ont été dénombrés 1203 Cooperia mûrs, soit, en moyenne, 86 par animal.

\section{Cooperia immatures}

Sur ces 69 zébus, 39 d'entre eux (34 traités et 5 témoins) se sont révélés, au grattage de la muqueuse intestinale, porteurs de Cooperia immatures à différents stades d'évolution. Ont été rencontrés des formes $L_{4}$, des formes $L_{5}$, des adultes immatures non gravides que l'on peut déterminer avec précision à partir des descriptions de STEWART (33) pour Cooperia punctata, d'HERLICH (21) et de KEITH (24) pour Cooperia pectinata, ainsi que des résultats obtenus en cultivant in vitro les mêmes Nématodes $(25,26)$.

Chronologiquement, après administration de 
larves $L_{3}$ à des animaux neufs $(J+0)$, l'évolution se fait ainsi :

\begin{tabular}{|c|c|c|}
\hline & $\begin{array}{l}\text { Cooperia } \\
\text { pectinata }\end{array}$ & $\begin{array}{l}\text { Cooperia } \\
\text { punctata }\end{array}$ \\
\hline Larves $\mathrm{L}_{\mathrm{j}}$ & $\mathbf{J}+0$ à $\mathbf{J}+3$ & $\mathbf{J}+0$ à $\mathbf{J}+4$ \\
\hline Mue $\mathrm{L}_{3} \rightarrow \mathrm{L}_{4}$ & $3^{e}$ jour & Fin du 4 e jour \\
\hline Larves $\mathbf{L}_{1}$ & $\mathrm{~J}+4$ à $\mathrm{J}+8$ & $\mathrm{~J}+5$ à $\mathrm{J}+7$ \\
\hline Mue $\mathrm{L}_{4} \rightarrow \mathrm{L}_{5}$ & $8 \mathrm{e}$ jour & $7 \mathrm{e}$ jour \\
\hline Larves $\mathrm{L}_{5}$ & $\mathbf{J}+9$ à $\mathbf{J}+12$ & $\mathrm{~J}+8$ à $\mathrm{J}+10$ \\
\hline Adultes & & \\
\hline non gravides & $12^{\mathrm{e}}$ jour & $10^{\mathrm{e}}$ jour \\
\hline Adultes gravides & $15 \mathrm{e}$ jour & $12 \mathrm{e}$ jour \\
\hline
\end{tabular}

Sur ces bases ont été recueillis :

- Chez 23 animaux, 126 larves $\mathrm{L}_{4}$ (en moyenne, 5,4 parasites par tête).

- Chez 18 animaux, 101 larves $\mathrm{L}_{5}$ (en moyenne, 5,6 parasites par tête).

- Chez 13 animaux, 179 adultes non gravides (en moyenne 13,7 parasites par tête).

De plus, dans l'intestin de seize animaux, il existait des formes immatures associées entre elles :

$-\mathbf{L}_{4}+\mathbf{L}_{5}, 4$ animaux.

- $\mathrm{L}_{4}+$ adultes non gravides, 4 animaux.

- $\mathrm{L}_{5}+$ adultes non gravides, 7 animaux.

- $\mathrm{L}_{5}+$ adultes gravides, 1 animal.

\section{DISCUSSION}

1. Une réinfestation après le 8 juillet, date du traitement est exclue, car les animaux traités n'hébergeaient - au bout de 72 heures plus aucun Cooperia adulte capable de pondre des œufs, donc de donner des larves infestantes $L_{3}$. De plus, les fumiers bourrés d'œufs de Némathodes ont été, durant cette période, retirés matin, midi et soir et soigneusement éliminés (19).

De même, entre le 3 et le 8 juillet, pendant la période d'observation, des précautions sévères ont été prises, de manière à éviter les recontaminations à partir d'œufs et de larves susceptibles d'évoluer accidentellement sur le sol (19).
La seule hypothèse valable est qu'il existait, au départ, dans le duodénum de plus de la moitié des animaux, une population mêlée composée :

- de Cooperia adultes gravides;

- de Cooperia sous forme de $\mathrm{L}_{4}$ inhibées.

Après traitement au Thiabendazole ou au Morantel, les Cooperia adultes et gravides sont détruits et disparaissent. Les formes $\mathrm{L}_{4}$ inhibées reprennent alors leur évolution qui les mènera au stade adulte. Le développement est irrégulier : 7 à 8 jours après le traitement, on ne rencontre que des $L_{4}$, puis leur nombre diminue peu à peu pour faire place à des $\mathrm{L}_{5}$, dans la proportion de cinq pour une le douzième jour.

Des femelles adultes sans œufs de Cooperia punctata * ont été isolées le neuvième jour après l'administration des médicaments et des femelles de Cooperia pectinata*, au même stade d'évolution, le dixième jour. Si l'on tient compte de la chronologie adoptée par STEWART (33) et par HERLICH (21), il ne s'agissait pas de femelles mûres ayant résisté à l'action des anthelminthiques et chez lesquelles la ponte aurait été arrêtée pendant quelques jours.

Le douzième jour, à l'autopsie, il est possible de mettre en évidence des femelles adultes de Cooperia pectinata renfermant quelques œufs qui ne sont décelables ni à la coproculture, ni à la coproscopie, ce qui confirme les observations d'HERLICH (21).

2. Le phénomène d'inhibition des larves de Trichostrongylidés est encore mal connu et, pour l'expliquer, on a fait intervenir de nombreux facteurs tels que la résistance de l'hôte, les réinfestations successives sur des pâturages contaminés, l'âge des animaux atteints et le nombre de larves ingérées.

Actuellement, les auteurs qui ont étudié ce problème pensent qu'il s'agit plutôt d'un phénomène de diapause, analogue à celui qui existe chez les Arthropodes (1, 5, 6, 7, 32). Dans les pays tempérés, en automne et en hiver, l'abaissement de la température et la diminution de la durée de l'ensoleillement influent sur les larves $\mathrm{L}_{3}$ d'Haemonchus, de Cooperia et d'Ostertagia. Celles-ci, parvenues dans les portions antérieures du tube digestif de leur

(*) Diagnose faite en mesurant les spicules des mâles inclus dans les lots de femelles. 
hôte, subissent une mue qui les mène au stade de larves $\mathbf{L}_{\boldsymbol{4}}$. Certaines d'entre elles évoluent normalement jusqu'au stade adulte. Les autres - souvent les plus nombreuses - sont inhibées au stade $L_{4}$ et cet arrêt de développement dont l'importance varie en fonction des réactions immunitaires individuelles des animaux atteints et des souches de parasites en cause $(2,28)$, dure tout l'hiver. "Il y a donc en quelque sorte adaptation biologique à la survie de l'espèce quand les conditions extérieures deviennent défavorables. "

Au printemps, le phénomène d'inhibition cesse spontanément, selon un processus que l'on ignore encore. Mais, certains facteurs, tels que l'humidité, la température, le changement d'alimentation au printemps avec un régime plus riche en vitamines, oligo-éléments et auxines, semblent jouer un rôle important.

C'est ce qui a été observé au cours de cette expérience : la reprise du développement des larves $\mathrm{L}_{4}$ inhibées, dans un pays sec et chaud, à climat bien tranché comme la République du Niger, paraît liée aux premières chutes de pluie et à l'apparition de l'hivernage en juillet. Dans le cas présent, jusqu'au 16 juillet 1974, les précipitations ont été peu nombreuses (deux) et de faible ampleur: seules, des larves $\mathrm{L}_{4}$ ont été rencontrées à l'autopsie des animaux. A partir du 17 juillet 1974, les tornades sont devenues plus violentes et plus fréquentes : l'évolution des larves inhibées s'est alors accélérée, à tel point que les 19 et 20 juillet, on dénombrait :

\begin{tabular}{|c|c|c|c|}
\hline & $\mathbf{L}_{*}$ & $\mathbf{L}_{5}$ & $\begin{array}{c}\text { Adultes } \\
\text { non gravides }\end{array}$ \\
\hline 19 juillet & 8,8 p. 100 & 52,9 p. 100 & 38,3 p. 100 \\
\hline 20 juillet & 0,6 p. 100 & 39,4 p. 100 & 60 p. 100 \\
\hline
\end{tabular}

Chez les témoins qui n'avaient reçu aucun médicament susceptible de modifier le comportement des larves $\mathrm{L}_{4}$, le phénomène est encore plus net. Quatre animaux sacrifiés le 21 juillet 1974 n'ont présenté qu'un nombre très faible de Cooperia à l'état de $\mathrm{L}_{5}$ ou d'adultes non gravides, soit au total 13 individus sur une population globale (adultes gravides compris) de 573 , soit 2,2 p. 100.

3. En Afrique, la présence de larves inhi- bées appartenant à diverses espèces de Trichostrongylidés a été observée chez les bovidés domestiques :

- En Afrique du Sud (30), de mars à novembre, dans une région sèche (Armoeds vlakte) où les précipitations ne dépassent pas 250-500 mm par an;

- En République du Tchad (18), entre le douzième et le treizième parallèle (pluviométrie 1954-1964: 476 à $780 \mathrm{~mm}$ ), de décembre à la mi-juin;

- Au Nigeria (20), sur le plateau de Bauchi, à 10 degrés de latitude Nord (pluviométrie : $1400 \mathrm{~mm}$ ). En fin de saison des pluies (octobre), les larves $\mathrm{L}_{\boldsymbol{4}}$ inhibées d'Haemonchus, de Trichostrongylus et de Cooperia sont mélangées aux formes adultes des mêmes Nématodes. De décembre à mars, ces dernières disparaissent peu à peu et sont remplacées par une nouvelle population issue des larves inhibées. Il en résulte que, les premiers jours d'avril (début de l'hivernage), les Trichostrongylus, les Haemonchus et, dans une moindre mesure, les Cooperia sont plus nombreux qu'en décembre.

\section{Conséquences}

Elles sont de deux ordres:

4.1. Les larves inhibées sont capables de reprendre leur évolution et de donner des vers adultes hématophages (Haemonchus) ou exerçant une forte action perturbatrice des métabolismes (Cooperia punctata et Cooperia pectinata), notamment celui des protides (22).

Ce passage coïncide avec la fin de la saison sèche, époque où les réserves alimentaires disponibles sont, en Afrique tropicale sèche, fortement réduites. L'animal, dont l'alimentation est insuffisante en quantité comme en qualité, résiste moins bien aux parasites, surtout lorsque plusieurs espèces sont associées entre elles : d'où des retards de croissance chez les jeunes, des pertes de poids et, parfois, de véritables enzooties de strongylose.

4.2. Les larves inhibées devenues adultes dispersent sur les pâturages un grand nombre d'œufs qui, en raison des conditions climatiques favorables (saison des pluies), donnent naissance, à leur tour, à des larves $\mathrm{L}_{3}$.

Celles-ci assurent l'infestation des jeunes animaux neufs, pleinement réceptifs, la réinfestation ou la surinfestation des ruminants qui ont déjà été en contact avec les mêmes parasites. 
Cette notion est importante en matière de prophylaxie. Il faut, en effet, attaquer et - si possible - détruire simultanément les larves inhibées et les adultes, ce qui implique le choix d'un médicament suffisamment efficace et la mise au point d'un calendrier de traitement précis.

Dans cet ordre d'idées, en Afrique centrale, deux séries de traitements sont actuellement recommandées :

- La première de septembre à novembre, selon les latitudes. Elle est destinée à réduire les populations de vers acquises au cours de la saison des pluies et de toucher un certain nombre de larves en état d'inhibition;

- la seconde de janvier à mai. Elle a pour but l'élimination :

a) Des Bunostomum et des Oesophagostomum adultes qui, de par leur biologie propre, sont alors abondants et sensibles à l'action des anthelminthiques habituels;

b) Des trichostrongles qui ont survécu au traitement précédent.

La sécheresse de l'air, les températures élevées et l'absence de précipitations empêchent l'évolution des cufs expulsés. La plupart des Nématodes ayant été chassés, les animaux résistent mieux à la disette alimentaire de la fin de la saison sèche et l'ensemencement massif et régulier des pâturages au début de la saison des pluies est beaucoup plus aléatoire.

\section{CONCLUSIONS}

1. Au cours d'essais thérapeutiques effectués au Laboratoire de Niamey (République du Niger) en juillet 1974,92 p. 100 des zébus autopsiés ont été trouvés porteurs de Cooperia pectinata et de Cooperia punctata, adultes et gravides. 52 p. 100 d'entre eux hébergeaient également des formes immatures $\left(L_{4}\right)$ en état d'inhibition.

Celles-ci reprennent le cours de leur développement normal au début de la saison des pluies (un peu plus de la mi-juillet, dans le cas de cette observation).

2. Ce phénomène d'inhibition que l'on peut assimiler au phénomène de diapause chez les Arthropodes existe non seulement dans les régions tempérées des deux hémisphères, mais encore dans certaines régions d'Afrique tropicale, entre le $10^{\text {e }}$ et le $14^{\text {e }}$ degré de latitude Nord.

3. Dans ces zones, les larves inhibées de Trichostrongylidés (Haemonchus, Cooperia, Trichostrongylus) devenues adultes représentent un danger certain pour la santé des animaux dont elles diminuent la résistance à une époque de soudure où les ressources alimentaires sont insuffisantes, souvent presque inexistantes.

4. En Afrique, les enquêtes épidémiologiques concernant les helminthoses du bétail doivent tenir compte de cette situation. Il en est de même pour les plans de prophylaxie et les calendriers de traitement.

\title{
SUMMARY
}

Inhibited development of Cooperia punctata and Cooperia pectinata larvae in zebu cattle of Niger

\begin{abstract}
The authors point out the presence, at the end of the dry season, of Cooperia pectinata and Cooperia punctata inhibited $\mathrm{L}_{4}$ larvae in the small intestine of zebu cattle slaughtered in Niamey (Niger).

The inhibited immature stages resume development at the onset of the rains.

The authors count the similar cases at present known in Africa and draw inference from that situation on the health of animals and the treatment of african bovine Trichostrongylosis.
\end{abstract}

\section{RESUMEN}

Inhibición del desarrollo de las larvas de Cooperia punctata y de Cooperia pectinata en el cebú de Niger

Los autores señalan la existencia al fin de la estación seca, de larvas $\mathrm{L}_{4}$ inhibidas de Cooperia punctata y de Cooperia pectinata en el intestino 
delgado de un poco más de 50 p. 100 de los cebues matados en la región de Niamey (Republica de Niger).

Desde el comienzo de las primeras lluvias, dichas larvas vuelven a coger su evolución normal.

Despues del censo de los casos similares actualmente conocidos en Africa, los autores consideran las consecuencias de esta situación sobre la salud de los animales y sobre el tratamiento de las estrongilosis en medio africano.

\section{BIBLIOGRAPHE}

1. ANDERSON (N.), ARMOUR (J.), RITCHIE (J. D.), URQUARTH (G. M.). Inhibited development of Ostertagia ostertagi. Vet. Rec., 1965, 77 (5) : $146-147$.

2. ARMOUR (J.), JENNINGS (F. W.), URQUARTH (G. M.). The possible existence of two strains of Ostertagia ostertagi. Vet. Rec., 1967, 80 (5) : 208-209.

3. ARMOUR (J.), JENNINGS (F, W.), URQUARTH (G. M.). Inhibition of Ostertagia ostertagi at the early fourth stage. I. The seasonal incidence. Res. vet. Sci., 1969, 10 (3): 232-237.

4. ARMOUR (J.), JENNINGS (F. W.), URQUARTH (G. M.). Inhibition of Ostertagia ostertagi at the early fourth stage. II. The influence of environment on host or parasite. Res. vet. Sci., 1969,10 (3) : 238-244.

5. ARMOUR (J.). Bovine Ostertagiosis: a review. Vet. Rec., 1970, 86 (7) : 184-190.

6. BLITZ (N.M.), GIBBS (H. C.). Studies on the arrested development of Haemonchus contortus in sheep. I. The induction of arrested development. Int. J. Parasit., 1972, 2 (1) : 5-12.

7. BLITZ (N. M.), GIBBS (H. C.). Studies on the arrested development of Haemonchus contortus in sheep. II. Termination of arrested development and the spring-rise phenomenon. Int. J. Parasit, 1972, 2 (1): 13-22.

8. BRUNDSON (R. V.). Inhibited development of Ostertagia spp. and Cooperia spp. in naturally acquired infections in calves. N.Z. vet. J., 1972, 20 (10): 183-189.

9. BRUNDSON (R. V.). Inhibited development of Haemonchus contortus in naturally acquired infections in sheep. $N . Z$. vet, J., 1973, $21(6): 125$ 126.

10. CONNAN (R. M.) The seasonal incidence of inhibition of development in Haemonchus contortus. Res. vet. Sci., 1971, 12 (3) : 272-274.

11. CONNAN (R.M.). Hyostrongylus rubidus: the size and structure of worm populations in adult pigs. Vet. Rec., 1971, 89 (7) : 186-191.

12. CONNAN (R. M.). Arrested development in Chabertia ovina. Res, vet. Sci., 1974, 16 (2) : 240-243.

13. DUNSMORE (J.D.). Retarded development of Ostertagia species in sheep. Nature, London, 1960 , 186 (4729) : 986-987.

14. EUZEBY (J.). Diagnostic expérimental des helminthoses animales. Paris, Vigot Frères, 1958.

15. FERNANDO (S. T.), SOULSBY (E. J. L.). Immunological studies of Mecistocirrus digitatus infection in calves. Res. vet. Sci., 1970, 11 (2): 175 182.

16. GEVREY (J.). Les formes libres des « Strongles digestifs " des ovins. Morphologie, culture all laboratoire, écologie. Thèse Doctorat es Sciences, Lyon, 1971.

17. GIBBS (H.C.). Transmission of parasites, with reference to Strongyles of domestic sheep and cattle. Can. J. Zool., 1973, 51 (2) : 281-289.

18. GRABER (M.). Etude du pouvoir anthelmin- thique du Tétramisole (16535 R.P.) sur divers helminthes du Zébu de la République du Tchad. Rev. Elev. Méd, vét. Pays trop., 1966, 19 (4): 511-526.

19. GRABER (M.), TAGER-KAGAN (P.). Essai de traitement du polyparasitisme du Zébu à l'aide des associations Disto-5 -Thiabendazole et Disto-5 Tartrate de Morantel. I.E.M.V.T,, 1974, ' T.I., 50 p.; T. II, 135 p.

20. HART (J.A.). Observations on the dry season infestations of Zebu cattle in Northern Nigeria. Brit. vet. J., 1964, 120 (2) : 87-95.

21. HERLICH (H.). The development of Cooperia pectinata, a Nematode parasite of cattle. $A \mathrm{~m} . J$. vet. Res., 1965, 26 (114): 1026-1031.

22. HERLICH (H.). The effects of intestine worms Cooperia pectinata and Cooperia oncophora on experimentally infected calves. Am. J. vet. Res, 1965, 26 (114) : 1032-1036.

23. HERLICH (H.). Effects of Cooperia pectinata on calves: two levels of repeated oral inoculation. Am. J. vet. Res., 1967, 28 (122) : 71-77.

24. KEITH (R. K.). The life-history of Cooperia pectinata, Ransom. Aust. J. Zool., 1967, 15 (4) : 739744.

25. LELAND Jr (S.E.). The in vitro development of Cooperia pectinata, a Nematode parasite of cattle from third stage larvae, including egg production. Parasitology, 1967, 53 (3) : 630-633.

26. LELAND $\mathrm{Jr}$ (S. E.). In vitro cultivation of Cooperia punctata from egg to egg. Parasitology, 1967, 53 (5) : 1057-1060.

27. MICHEL (J. F.), LANCASTER (M. B.), HONG (C.). Observations on the inhibition of Cooperia Oncophora in calves. Brit. vet. J., 1970, 126: XXXV-XXXVII.

28. MICHEL (J. F.), LANCASTER (M. B.), HONG (C.). Inhibition of development: variation within a population of Ostertagia ostertagi. J. comp. Path. Ther., 1973, 83 (3) : 351-356.

29. REID (J.F.S.), ARMOUR (J.). Seasonal fluctuation and inhibited development of gastrointestinal Nematodes of sheep. Res. vet. Sci., 1972, 13 (3) : 225-229.

30. REINECKE (R.K.). A field study of some Nematode parasites of bovines in a semiarid area, with special reference to their biology and possible methods of prophylaxis. Onderstepoort $\mathrm{J}$. vet. Res., 1960, 28 (3) : 365-464.

31. ROSS (J. G.). The seasonal incidence of ostertagiosis in cattle in Northern Ireland. Vet. Rec., 1965, 77 (1) : 16-19.

32. SMMH (H. J.). Inhibited development of Ostertagia ostertogi, Cooperia oncophora and Nematodirus helvetianus in parasite-free calves grazing fall pastures. Am. J. vet. Res., 1974, 35 (7): 935938.

33. STEWART (T. B.). The life-history of Cooperia punctata, a Nematode parasite in cattle. Parasitology, 1954, 40 (3) : 321-327. 